Robust estimation of intraweek periodicity in volatility and jump detection

K. Boudt, C. Croux and S. Laurent

DEPARTMENT OF DECISION SCIENCES AND INFORMATION MANAGEMENT (KBI) 


\title{
Robust estimation of intraweek periodicity in volatility and jump detection
}

\author{
Kris Boudt* Christophe Croux ${ }^{\dagger} \quad$ Sébastien Laurent ${ }^{\ddagger}$
}

\begin{abstract}
Opening, lunch and closing of financial markets induce a periodic component in the volatility of high-frequency returns. We propose a non-parametric weighted standard deviation and parametric truncated maximum likelihood estimation procedure for the periodic component in volatility and show that they are robust to price jumps. We also show that robust periodicity estimates can be used to increase the accuracy of jump detection methods. We compare the classical and robust methods for the 5-minute EUR/USD returns. The robust intraweek periodicity estimates are lower than the classical ones on Tuesday-Friday 8:30-8:35 EST and Monday-Friday 10:00-10:05 EST. The higher values for the non-robust estimates are likely to be due to jumps. Accounting for the periodicity in the volatility of high-frequency returns is especially important to detect the relatively small jumps occurring at times for which volatility is periodically low and to reduce the number of spurious jump detections at times of periodically high volatility.
\end{abstract}

Keywords: high-frequency data; jump detection; periodicity; robust statistics.

${ }^{*}$ Faculty of Business and Economics, K.U.Leuven and Lessius University College.

${ }^{\dagger}$ Faculty of Business and Economics, K.U.Leuven.

${ }^{\ddagger}$ CeReFiM, University of Namur and CORE.

Correspondence to: Kris Boudt, Faculty of Business and Economics, 69 Naamsestraat, B3000 Leuven, Belgium. E-mail: Kris.Boudt@econ.kuleuven.be. Tel: +32 16326728 Fax: $+3216326624$

We thank Charles Bos, Dobrislav Dobrev, Geert Dhaene, Tatyana Krivobokova, Jerome Lahaye, Alfio Marazzi, Mattias Salibián-Barrera and participants of the 6th OxMetrics conference for helpful suggestions. Financial support from the FWO, Research Fund K.U.Leuven and Belgian Program on Interuniversity Poles of Attraction initiated by the Belgian State Prime Minister's Office, science policy programming, is gratefully acknowledged. The usual disclaimer applies. 


\section{Introduction}

Price jumps and the periodic behavior of intraday volatility due to opening, lunch and closing of financial markets are salient features of high-frequency price series. Andersen and Bollerslev (1997) and Andersen et al. (2007) document the importance of allowing for jumps and for periodicity of intraday volatility in the non-parametric estimation and forecasting of volatility, but treat these two features separately.

Neglecting the potential presence of jumps when estimating the periodic component of intraday volatility can cause a large estimation bias. Andersen et al. (2001) illustrate that removing one observation from the sample completely changes the estimated volatility pattern reported by Ito et al. (1998). Andersen et al. (2007) note that jump detection based on comparing standardized intraday returns with critical values of the normal distribution "will tend to over-reject the diffusive null hypothesis whenever there is substantial intraday variation in volatility".

The contribution of this paper is threefold. First of all, we define estimators for the periodic component in intraday volatility that are robust to price jumps. These estimators are robustifications of Andersen and Bollerslev (1997) and Taylor and Xu (1997)'s periodicity estimators. Robust estimates of the periodicity in intraday volatility are useful for a broad range of applications. Examples include the study of the effect of macroeconomic news (Andersen and Bollerslev, 1998b) and of intraday volume (Goodhart and O'Hara, 1997) on intraday volatility, forecasting of intraday variances (Taylor and $\mathrm{Xu}, 1997$ ) and intraday jump detection (Andersen et al., 2007; Lee and Mykland, 2008). Accurate detection of the returns affected by jumps is needed to disentangle the information in the high-frequency returns affected by jumps and the returns corresponding to the small, erratic price movements. These tests have been used for the estimation of the daily integrated variance of a log-price diffusion with jumps (Boudt et al., 2008b) and testing for the semi-martingale assumption (Andersen et al., 2007).

Our second contribution is to show that the robust periodicity estimate 
can be used to increase the accuracy of the non-parametric jump detection methods proposed by Andersen et al. (2007) and Lee and Mykland (2008). Both methods identify the returns affected by jumps by comparing each standardized return with a given threshold. The standardized return equals the absolute value of the tested return divided by a robust estimate of its standard deviation based on the returns observed in a window around that return. Calendar-linked events such as opening, lunch and closing of the financial markets around the world cause a strong periodic variation of volatility within the local window. We show that in the presence of such a periodic pattern in intraday volatility, the original parametric and non-parametric tests overdetect (resp. underdetect) jumps at the intraday times for which volatility is periodically high (resp. low). Andersen et al. (2007) recognize this and, as a robustness check, they repeat their analysis for the returns from which periodicity has been filtered away. However, their periodicity estimator is very sensitive to jumps. We propose the robustly filtered jump test statistic which equals the original jump test statistic divided by an estimate of the periodicity component in intraday volatility that is highly robust to jumps. The simulation study confirms that the robustly filtered jump test has better finite sample properties than the original test in the presence of periodic time-variation in the volatility of the high-frequency returns.

Finally, the paper contributes to the empirical literature on periodicity in intraday volatility (see e.g. Andersen and Bollerslev 1998b; Martens et al. 2002 ) and price jumps (see e.g. Bos 2008; Lahaye et al. 2007; Lee and Mykland 2008). We compare the proposed robust estimation and jump detection procedures with the standard ones for the 5-minute EUR/USD returns. We find large differences between the non-robust and robust periodicity estimates for the Sunday evening trading intervals and the intervals during which macroeconomic news is usually released. We attribute these differences to the presence of jumps, because these are the intervals for which a higher probability of jump occurrences is expected. We also compare the results of jump detection using the original and the filtered tests. We find that these two tests detect a similar proportion of returns affected by jumps, but the filtered tests detect less jumps 
at times of high periodicity and more jumps at times of low periodicity. From a market microstructure perspective, this makes sense since periodically low volatility is associated with periodically low volume. Since jumps are more likely to occur when markets are less liquid (Farmer et al., 2004), we expect more jumps at intraday times of periodically low volatility.

The remainder of the paper is organized as follows. Section 2 introduces the robust periodicity estimators and studies their properties. Section 3 first recalls the non-parametric jump detection techniques proposed by Andersen et al. (2007) and Lee and Mykland (2008) and then shows how the robust periodicity estimates can be used to make these tests more accurate in the presence of a periodic variation in intraday volatility. Section 4 applies the new method to the 5-minute EUR/USD return series. Finally, Section 5 summarizes our conclusions and outlines some implications for further research.

\section{Robust estimation of intraweek periodicity}

This section proposes robust estimators for the intraweek periodic pattern in the volatility of high-frequency return series. We suppose that our sample consists of $T$ days of $M$ equally-spaced and continuously compounded intraday return observations $r_{i}(i=1, \ldots, M T)$ of a financial asset. As usual when dealing with intraday data, we exclude overnight returns from the analysis. We normalize the length of one trading day to unity such that $\Delta=1 / M$ equals the time elapsed between two consecutive return observations. Hence, $r_{i}$ equals the return over the time interval $[(i-1) \Delta, i \Delta]$. We write these returns as the discrete changes of the underlying continuous-time log-price process, i.e. $r_{i}=p(i \Delta)-p((i-1) \Delta)$. Subsections 2.2 and 2.3 propose robust estimators for the periodicity in the volatility of the $r_{i}$ 's. These estimators are consistent under the model presented in Subsection 2.1. 


\subsection{Model}

We assume that the log-price process $p(s)$ follows a Brownian SemiMartingale with Finite Activity Jumps (BSMFAJ) diffusion. Andersen et al. (2007) and Lee and Mykland (2008) use the same model. Under the BSMFAJ model the log-price follows a diffusion consisting of the sum of a conditionally normal random process with mean $\mu(s) d s$ and variance $\sigma^{2}(s) d s$, and of a jump generating process. The occurrence of jumps is governed by a finite activity counting process $q(s)$ and the size of the jumps is given by $\kappa(s) .{ }^{1}$ Let $w(s)$ be a standard Brownian motion, then a BSMFAJ log-price diffusion admits the following representation

$$
\text { BSMFAJ: } \quad d p(s)=\mu(s) d s+\sigma(s) d w(s)+\kappa(s) d q(s) .
$$

Throughout, we will be operating with sufficiently high-frequency return series such that the mean process can be safely ignored. Thus, for simplicity, we set $\mu(s)=0$ in the sequel. We also assume that the spot volatility process is continuous. Under the BSMFAJ model, we have that, for small values of $\Delta$, the returns $r_{i}$ in an interval without jumps in the underlying price diffusion process, are conditionally normally distributed with mean zero and variance $\sigma_{i}^{2}=\int_{(i-1) \Delta}^{i \Delta} \sigma^{2}(s) d s$.

As shown by Andersen and Bollerslev (1998b), the spot volatility at time $s$ is well approximated by the average volatility in a window around $s$, multiplied with a factor that corrects for the diurnal pattern in volatility. We formalize this as follows.

First of all, consider a division of $[0, T]$ in time intervals of length $\lambda$, called "local windows". As such, the $M T$ observations are divided in groups of $\lfloor\lambda / \Delta\rfloor$ contiguous observations. For descriptive studies of periodicity in intraday volatility of exchange rate data such as the 5-minute EUR/USD returns, it is common to set $\lambda$ to one day (Andersen and Bollerslev, 1997, 1998b). For intraday jump detection based on the absolute return divided by a robust estimate of the scale of the returns in a local window of length $\lambda$ (Andersen et al.,

\footnotetext{
${ }^{1} \mathrm{~A}$ count process is defined to be of finite activity if the change in the count process over any interval of time is finite with probability 1 .
} 
2007; Lee and Mykland, 2008), the window length depends on the frequency at which the returns are observed and the persistency of the variance of the standardized returns. While for intraday jump detection using tick by tick data of a liquid financial asset $\lambda$ can be as small as 15 minutes, intraday jump detection using 5 or 15 -minute returns requires a $\lambda$ of one day.

Secondly, we consider the time transformation $\tau(s)$ indicating the position of $s$ in the periodicity cycle

$$
\tau(s)=s \bmod L,
$$

with mod the modulo operator. ${ }^{2}$ We suppose that the cycle repeats itself every $L$ days and thus $\tau(s)=\tau(s+L)$, for any $s$. Common choices for $L$ are unity (the cycle repeats itself every day) or five (the cycle repeats itself every week of five trading days). Without loss of generality, we assume that the number of windows per cycle, i.e. $L / \lambda$, is integer.

We then define the periodicity factor at time $s$ and for windows of length $\lambda$ as the square root of the expected value of the ratio between the spot variance and the mean variance over the window, conditional on the position of $s$ in the cycle.

Definition 1 The periodicity factor $f_{\lambda}$ at time $s \in[(l-1) \lambda, l \lambda]$ is defined as

$$
f_{\lambda}^{2}(\tau(s)) \equiv E\left[\frac{\sigma^{2}(s)}{\lambda^{-1} \int_{(l-1) \lambda}^{l \lambda} \sigma^{2}(s) d s} \mid \tau(s)\right] .
$$

The periodity factor $f_{\lambda}(\tau(s))$ is above one at times $\tau(s)$ for which volatility is periodically high and below one if volatility is periodically low.

Remark 1 The periodicity factor depends on the length of the local window. For simplicity, we will omit the index $\lambda$ whenever no confusion is possible and denote

$$
f_{i} \equiv f_{\lambda}(\tau(i \Delta))
$$

Remark 2 Since $f_{\lambda}^{2}(\tau(s))$ is defined as the expectation of the ratio between the spot variance and the mean variance over the window, conditional on $\tau(s)$,

\footnotetext{
${ }^{2}$ The modulo operation $a \bmod b$ returns the remainder after integer division of $a$ by $b$.
} 
the mean of $f_{\lambda}^{2}(\tau(s))$ over the whole local window is unity, i.e.

$$
\int_{(l-1) \lambda}^{l \lambda} f_{\lambda}^{2}(\tau(s)) d s=1 .
$$

Denote by $N_{i}$ the collection of indices $j$ that belong to the same window as $i$. The finite sample counterpart of (2.5) is that

$$
\frac{1}{\lfloor\lambda / \Delta\rfloor} \sum_{j \in N_{i}} f_{j}^{2}=1 .
$$

In the estimation, we impose the standardization condition (2.6), which guarantees that the squared periodicity factor has mean one over the local window. Similar standardization conditions have been considered in the literature. Taylor and Xu (1997) impose that the mean squared periodicity factor equals one over the whole cycle and not only over the local window. Andersen and Bollerslev (1997) impose that the mean of the periodicity factor (and not the squared periodicity factor) equals one over the day.

Our robust estimators for $f_{\lambda}(\tau(s))$ are based on the assumption that for small values of $\lambda$, the spot volatility $\sigma(s)$ can be rewritten as a locally constant process, multiplied with the periodicity factor.

Assumption 1 Let $\tilde{\sigma}_{\lambda}^{2}(l)=\lambda^{-1} \int_{(l-1) \lambda}^{l \lambda} \sigma^{2}(s) d s$. We assume that for all $s \in$ $[(l-1) \lambda, l \lambda]: \sigma(s)=\tilde{\sigma}_{\lambda}(l) f_{\lambda}(\tau(s))+\mathrm{O}_{p}(\lambda)$.

Assumption 1 requires that the spot volatility after filtering out the periodicity is approximately constant over the local window. This is less restrictive than Lee and Mykland (2008)'s assumption that the spot volatility without filtering out the periodicity is approximately constant over the local window. ${ }^{3}$

The value $s_{i}=\sigma_{i} / f_{i}$ is the periodically adjusted volatility of $r_{i}$. By Assumption $1, s_{i}$ is approximately constant within each local window, provided

\footnotetext{
${ }^{3}$ Our definition of windows is slightly different than in Lee and Mykland (2008), where the window equals the time period of length $\lambda$ that immediately precedes the return for which the presence of jumps is tested. The exact definition of the window does not matter as long as it is reasonable to assume that $\sigma_{i}^{2} / f_{i}^{2}$ is approximately constant within the local window.
} 
the window is sufficiently short. Denote by $r_{j+1}, \ldots, r_{j+\lfloor\lambda / \Delta\rfloor}$ the $\lfloor\lambda / \Delta\rfloor$ returns in a local window of length $\lambda$ around $r_{i}$. We estimate $s_{i}$ using the square root of a normalized version of Barndorff-Nielsen and Shephard (2004)'s realized bipower variation over the local window

$$
\hat{s}_{i}=\sqrt{\frac{\pi}{2} \frac{1}{\lfloor\lambda / \Delta\rfloor-1} \sum_{l=j+2}^{j+\lfloor\lambda / \Delta\rfloor}\left|r_{l}\right|\left|r_{l-1}\right| .}
$$

In Subsection 2.4 we provide assumptions under which, for $\lambda \rightarrow 0$ and $\lambda / \Delta \rightarrow \infty$ and if $r_{i}$ is not affected by jumps, the standardized high-frequency return

$$
\bar{r}_{i}=r_{i} / \hat{s}_{i}
$$

is conditionally normally distributed with mean zero and variance equal to the squared periodicity factor. This result suggests to estimate the periodicity factor using either a non-parametric or parametric estimator of the scale of the standardized returns $\bar{r}_{i}$. Such an estimator has to be robust to price jumps.

\subsection{Non-parametric estimation of periodicity}

The non-parametric periodicity estimator is based on a scale estimate of the standardized returns that share the same periodicity factor. Let $\bar{r}_{1, i}, \ldots, \bar{r}_{n_{i}, i}$ be the set of standardized returns having the same periodicity factor as $\bar{r}_{i}$. If the periodicity factor depends only on the time of the day and day of the week at which $r_{i}$ is observed, we have that $\bar{r}_{1, i}, \ldots, \bar{r}_{n_{i}, i}$ are the returns observed on the same time of the day and day of the week as $r_{i}$. As in Andersen and Bollerslev (1997), one could also consider the calendar effects jointly with macroeconomic news effects. Then $\bar{r}_{1, i}, \ldots, \bar{r}_{n_{i}, i}$ are the returns observed on the same time of the day and day of the week as $r_{i}$, on a day with the same macroeconomic news releases as for $r_{i}$.

The non-parametric periodicity estimator proposed by Taylor and Xu (1997) is based on the Standard Deviation (SD) of all standardized returns belonging to the same local window as $\bar{r}_{i}$, i.e. $\mathrm{SD}_{i}=\sqrt{\frac{1}{n_{i}} \sum_{j=1}^{n_{i}} \bar{r}_{j, i}^{2}}$ The SD periodicity 
estimator equals

$$
\hat{f}_{i}^{\mathrm{SD}}=\frac{\mathrm{SD}_{i}}{\sqrt{\frac{1}{\lfloor\lambda / \Delta\rfloor} \sum_{j \in N_{i}} \mathrm{SD}_{j}^{2}}} .
$$

The denominator in (2.9) ensures that the standardization condition (2.6) is met.

In the absence of jumps, the SD is efficient since the standardized returns are normally distributed. In the presence of jumps, the SD estimator is strongly biased, since it suffices that one observation in the sample is affected by a jump to make the periodicity estimate extremely large. Our proposal is to replace the standard deviation in (2.9) by a robust estimator.

Amongst the large number of robust scale estimators available in the literature (see Maronna et al., 2006, for an overview), we recommend the use of the Shortest Half scale estimator proposed by Rousseeuw and Leroy (1988). It has the property of being, among a wide class of scale estimators, the estimator for which jumps can cause the smallest maximum bias possible (Martin and Zamar, 1993). Under normality, it has the same efficiency as the median absolute deviation and the interquartile range. For the definition of the Shortest Half (ShortH) scale estimator, we need the corresponding order statistics $\bar{r}_{(1), i}, \ldots, \bar{r}_{\left(n_{i}\right), i}$ such that $\bar{r}_{(1), i} \leq \bar{r}_{(2), i} \leq \ldots \leq \bar{r}_{\left(n_{i}\right), i}$. The shortest half scale is the smallest length of all "halves" consisting of $h_{i}=\left\lfloor n_{i} / 2\right\rfloor+1$ contiguous order statistics. These halves equal $\left\{\bar{r}_{(1), i}, \ldots, \bar{r}_{\left(h_{i}\right), i}\right\}, \ldots,\left\{\bar{r}_{\left(n_{i}-h_{i}+1\right), i}, \ldots, \bar{r}_{\left(n_{i}\right), i}\right\}$, and their length is $\bar{r}_{\left(h_{i}\right), i}-\bar{r}_{(1), i}, \ldots, \bar{r}_{\left(n_{i}\right), i}-\bar{r}_{\left(h_{i}\right), i}$, respectively. The corresponding scale estimator (corrected for consistency under normality) equals the minimum of these lengths

$$
\mathrm{ShortH}_{i}=0.741 \cdot \min \left\{\bar{r}_{\left(h_{i}\right), i}-\bar{r}_{(1), i}, \ldots, \bar{r}_{\left(n_{i}\right), i}-\bar{r}_{\left(n_{i}-h_{i}+1\right), i}\right\} .
$$

Analogous to the SD estimator in (2.9), the ShortH estimator for the periodicity factor of $r_{i}$ equals

$$
\hat{f}_{i}^{\text {ShortH }}=\frac{\text { ShortH }_{i}}{\sqrt{\frac{1}{\lfloor\lambda / \Delta\rfloor} \sum_{j \in N_{i}} \text { ShortH }_{j}^{2}}} .
$$

The ShortH is highly robust to jumps, but it has only a $37 \%$ efficiency under normality of the $\bar{r}_{i}$ 's (Rousseeuw and Leroy, 1988). A better trade-off 
between the efficiency of the SD under normality and the high robustness to jumps of the ShortH is obtained using a Weighted Standard Deviation (WSD), where the weights depend on the value of the standardized return divided by the ShortH periodicity estimate

$$
\hat{f}_{i}^{\mathrm{WSD}}=\frac{\mathrm{WSD}_{i}}{\sqrt{\frac{1}{\lfloor\lambda / \Delta\rfloor} \sum_{j \in N_{i}} \mathrm{WSD}_{j}^{2}}},
$$

with

$$
\mathrm{WSD}_{j}=\sqrt{1.081 \cdot \frac{\sum_{l=1}^{n_{j}} w_{l, j} \bar{r}_{l, j}^{2}}{\sum_{l=1}^{n_{j}} w_{l, j}}} .
$$

The weights are given by $w_{l, j}=w\left(\bar{r}_{l, j} / \hat{f}_{j}^{\text {ShortH }}\right)$ where we use as a weight function

$$
w(z)= \begin{cases}1 & \text { if } z^{2} \leq 6.635 \\ 0 & \text { else. }\end{cases}
$$

The threshold 6.635 equals the $99 \%$ quantile of the $\chi^{2}$ distribution with one degree of freedom. The WSD in (2.13) has a $69 \%$ efficiency under normality of the $\bar{r}_{i}$ 's, as apposed to the $37 \%$ efficiency of the ShortH (see Boudt et al., 2008a, for details).

\subsection{Parametric estimation of periodicity}

The non-parametric estimators for the periodic component of intraday volatility use only the subset of the data for which the returns have the same periodicity factor. Andersen and Bollerslev (1997) show that more efficient estimates can be obtained if the whole time series dimension of the data is used for the estimation of the periodicity process. They use the result that, in the absence of jumps, the standardized returns are normally distributed with mean zero and variance $f_{i}^{2}$. They consider the regression equation

$$
\log \left|\bar{r}_{i}\right|-c=\log f_{i}+\varepsilon_{i}
$$

where the error term $\varepsilon_{i}$ is i.i.d. distributed with mean zero and having the density function of the centered absolute value of the log of a standard normal 
random variable, i.e.

$$
g(z)=\sqrt{2 / \pi} \exp [z+c-0.5 \exp (2(z+c))]
$$

The parameter $c=-0.63518$ equals the mean of the $\log$ of the absolute value of a standard normal random variable. Andersen and Bollerslev (1997) then propose to model $\log f_{i}$ as a linear function of a vector of variables $x_{i}$ (such as sinusoid and polynomial transformations of the time of the day)

$$
\log f_{i}=x_{i}^{\prime} \theta_{*}
$$

with $\theta_{*}$ the true parameter value. Combining (2.14) with (2.16), we obtain the following regression equation

$$
\log \left|\bar{r}_{i}\right|-c=x_{i}^{\prime} \theta_{*}+\varepsilon_{i}
$$

It is common to estimate the parameter $\theta_{*}$ in $(2.17)$ by the OLS estimator. This approach is not efficient, since the error terms are not normally distributed. The efficient estimator is the maximum likelihood estimator. Denote $\rho^{\mathrm{OLS}}(z)=z^{2}$ and let $\rho^{\mathrm{ML}}(z)$ be the negative log likelihood function

$$
\rho^{\mathrm{ML}}(z)=-0.5 \log (2 / \pi)-z-c+0.5 \exp (2(z+c)) .
$$

The OLS and ML estimates equal

$$
\hat{\theta}^{\mathrm{OLS}}=\operatorname{argmin}_{\theta} \frac{1}{M T} \sum_{i=1}^{M T} \rho^{\mathrm{OLS}}\left(\varepsilon_{i, \theta}\right) \quad \text { and } \quad \hat{\theta}^{\mathrm{ML}}=\operatorname{argmin}_{\theta} \frac{1}{M T} \sum_{i=1}^{M T} \rho^{\mathrm{ML}}\left(\varepsilon_{i, \theta}\right),
$$

with $\varepsilon_{i, \theta}=\log \left|\bar{r}_{i}\right|-c-x_{i}^{\prime} \theta$. These $\rho$-functions are called loss functions and are plotted in Figure 1. The non-robustness of the OLS and ML estimators to jumps is due to the unbounded effect an observation can have on their loss function. In the simulation study of Subsection 2.5 we find that in particular the ML estimator has a large bias in the presence of jumps. Martens et al. (2002) mention that the effect of jumps on the OLS estimator is attenuated because the regression is based on the log of the standardized returns, but solely a log-transformation is not sufficient to attain robustness to jumps. 
Figure 1: Loss functions associated to the OLS and ML estimators. The horizontal line denotes the likelihood threshold and the vertical lines the upper and lower truncation levels based on the $99 \%$ quantile, used by the TML estimator.

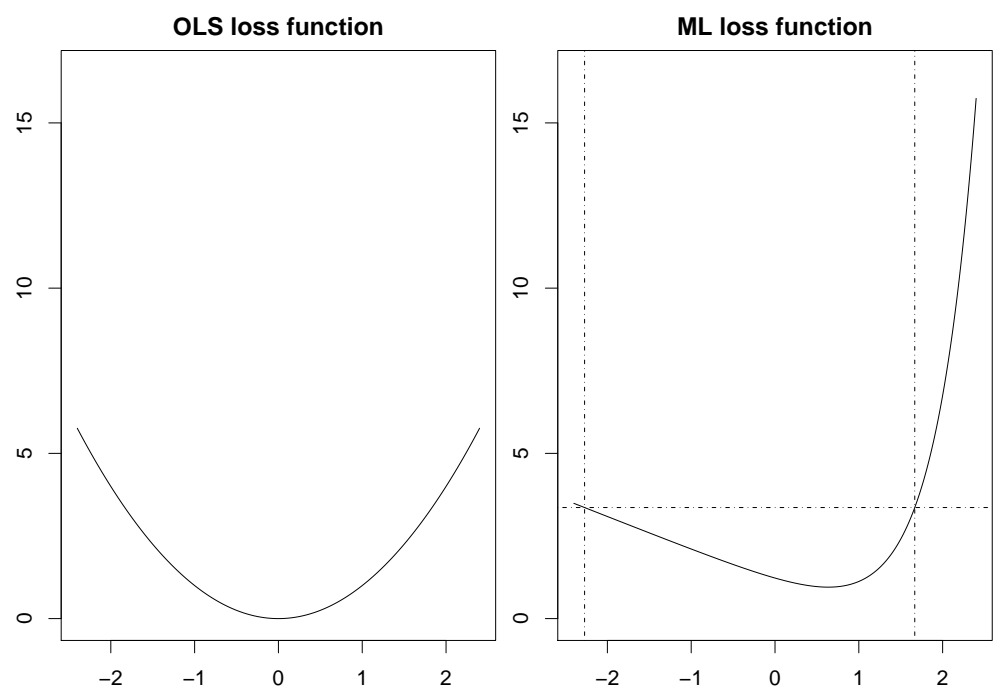

As an alternative to the OLS and ML estimators, we propose to use the Truncated Maximum Likelihood (TML) estimator introduced by Marazzi and Yohai (2004). This estimator gives a zero weight to observations that are outliers according to the value of the ML loss function. In a first step the residuals are computed using the robust non-parametric estimator $\hat{f}^{\mathrm{WSD}}$ in (2.12). Let

$$
e_{i}^{\mathrm{WSD}}=\log \left|\bar{r}_{i}\right|-c-\log \hat{f}_{i}^{\mathrm{WSD}} .
$$

Observations for which $\rho^{\mathrm{ML}}\left(e_{i}^{\mathrm{WSD}}\right)$ is large, have a low likelihood and are therefore likely to be outliers. Denote $q$ an extreme upper quantile of the distribution of $\varepsilon_{i}$. The TML estimator is defined as

$$
\hat{\theta}^{\mathrm{TML}}=\operatorname{argmin}_{\theta} \frac{1}{\sum_{i=1}^{M T} w_{i}} \sum_{i=1}^{M T} w_{i} \rho^{\mathrm{ML}}\left(\varepsilon_{i, \theta}\right),
$$


with

$$
w_{i}= \begin{cases}1 & \text { if } \rho^{\mathrm{ML}}\left(e_{i}^{\mathrm{WSD}}\right) \leq \rho^{\mathrm{ML}}(q) \\ 0 & \text { else. }\end{cases}
$$

The truncation on the basis of the value of the ML-function is illustrated in Figure 1 for the $99.5 \%$ quantile. All observations with $\rho^{\mathrm{ML}}\left(e_{i}^{\mathrm{WSD}}\right)>3.36$ receive a zero weight in the objective function of the TML estimator.

Like for the non-parametric periodicity estimators, we impose that the squared periodicity factor has mean one in the local window. The parametric estimate for the periodicity factor thus equals

$$
\hat{f}_{i}^{\mathrm{TML}}=\frac{\exp \left(x_{i}^{\prime} \hat{\theta}^{\mathrm{TML}}\right)}{\sqrt{\frac{1}{[\lambda / \Delta]} \sum_{j \in N_{i}} \exp \left(x_{i}^{\prime} \hat{\theta}^{\mathrm{TML}}\right)}},
$$

and similarly for $\hat{f}_{i}^{\mathrm{OLS}}$ and $\hat{f}_{i}^{\mathrm{ML}}$.

\section{$2.4 \quad$ Asymptotic properties}

This subsection presents the properties of the robust periodicity estimators under the assumption that the log-price series follows the BSMFAJ diffusion in (2.1). We derive our results for $\mu(s)=0$ but by Girsanov's theorem they also apply for the nonzero drift case, as in Zhang et al. (2005). Consistency of the periodicity estimators depends on the length of the local window $(\lambda)$ and the number of observations in that window $(\lfloor\lambda / \Delta\rfloor)$. We need on the one hand that we have a sufficiently large number of observations in the local window $(\lambda / \Delta \rightarrow \infty)$ and, on the other hand, that (after filtering the periodicity) the change in the volatility process is negligible for $\lambda \rightarrow 0$ (see Assumption 1). To prove consistency of the robust periodicity estimators in the presence of finite activity jumps, we further require that the total number of jumps occurring at the same intra-cycle time $\tau$ is finite. Then, if the total number of days $T \rightarrow \infty$, the proportion of returns affected by a jump for a given intraday time converges to zero.

Assumption 2 For any $i$, let $D_{i}$ be the set of indices $j$ for which $\tau(j \Delta)=$ $\tau(i \Delta)$. We assume that $\sup _{i} \sum_{j \in D_{i}}[q(j \Delta)-q((j-1) \Delta)]=\mathrm{O}_{p}(1)$. 
Write now $\bar{r}(i \Delta)=\bar{r}_{i}, \hat{f}^{\mathrm{SD}}(\tau(i \Delta))=\hat{f}_{i}^{\mathrm{SD}}, \hat{f}^{\mathrm{ShortH}}(\tau(i \Delta))=\hat{f}_{i}^{\mathrm{ShortH}}, \hat{f}^{\mathrm{WSD}}(\tau(i \Delta))=$ $\hat{f}_{i}^{\mathrm{WSD}}$ and $x(\tau(i \Delta))=x_{i}$ (these quantities are defined in Subsections 2.2-2.3). We first present results on the consistency of the SD, ShortH and WSD estimators under the BSM model, which is the BSMFAJ model without jumps. Proofs are outlined in the Appendix.

Result 1 Under the BSM model with spot volatility process satisfying Assumption 1, we have that $\hat{f}^{S D}(\tau(s))-f(\tau(s))$, $\hat{f}^{\text {Short } H}(\tau(s))-f(\tau(s))$ and $\hat{f}^{W S D}(\tau(s))-$ $f(\tau(s))$ converge in probability to 0 for $\lambda \rightarrow 0, \lambda / \Delta \rightarrow \infty$ and $T \rightarrow \infty$.

Result 2 shows that the robust non-parametric estimators $\hat{f}^{\mathrm{ShortH}}(s)$ and $\hat{f}^{\mathrm{WSD}}(s)$ are also consistent estimators under the BSMFAJ model.

Result 2 Under the BSMFAJ model with spot volatility process satisfying Assumption 1 and with jump occurrence process satisfying Assumption 2, we have that $\hat{f}^{S h o r t H}(\tau(s))-f(\tau(s)) \stackrel{p}{\rightarrow} 0$ and $\hat{f}^{W S D}(\tau(s))-f(\tau(s)) \stackrel{p}{\rightarrow} 0$ for $\lambda \rightarrow 0$, $\lambda / \Delta \rightarrow \infty$ and $T \rightarrow \infty$.

A final set of results is that the estimator $\hat{\theta}^{\mathrm{TML}}$ is consistent for $\theta_{*}$ under the BSM (Result 3) and BSMFAJ (Result 4) models, if the parametric specification of the periodicity function is correct.

Result 3 Under the BSM model with spot volatility process satisfying Assumption 1 , and if $\log f(\tau(s))=\theta_{*}^{\prime} x(\tau(s)), \hat{\theta}^{T M L} \stackrel{p}{\rightarrow} \theta_{*}$ for $\lambda \rightarrow 0, \lambda / \Delta \rightarrow \infty$ and $T \rightarrow \infty$.

Result 4 Under the BSMFAJ model with spot volatility process satisfying Assumption 1 and with jump occurrence process satisfying Assumption 2, and if $\log f(\tau(s))=\theta_{*}^{\prime} x(\tau(s)), \hat{\theta}^{T M L} \stackrel{p}{\rightarrow} \theta_{*}$ for $\lambda \rightarrow 0, \lambda / \Delta \rightarrow \infty$ and $T \rightarrow \infty$.

\subsection{Simulation study}

In this section we use simulated data to evaluate the effect of jumps on the bias and efficiency of the periodicity estimators to jumps. Let $w(s)$ and $b(s)$ be two independent Brownian motions. We generate 5-minute returns from 
the BSMFAJ price diffusion in $(2.1)$ with $\mu(s)=0$ and $\sigma(s)$ specified as a multiplicative process of the periodicity function $f(\tau(s))$, which depends only on the time of the day $\tau(s)=s-\lfloor s\rfloor$, and a GARCH diffusion process, i.e.

$$
\sigma(s)=f(s-\lfloor s\rfloor) \sigma_{\text {garch }}(s) .
$$

The GARCH diffusion is calibrated at the values implied by the GARCH estimates obtained by Andersen and Bollerslev (1998a) for the daily returns on the Deutschemark-US Dollar exchange rates from 1987 until 1992

$$
d \sigma_{\text {garch }}^{2}(s)=-.035\left[\sigma_{\text {garch }}^{2}(s)-.636\right] d s+.144 \sigma_{\text {garch }}^{2}(s) d b(s) .
$$

The function $f(\tau(s))$ used in the simulation is plotted in dashed line in Figure 2. It is based on $\log f(\tau(s))=\theta^{\prime} x(\tau(s))$ with $x(\tau(s))$ a vector holding quadratic and sinusoid transformations of $\tau(s)$. The vector $\theta$ is calibrated at its TML estimate for the January 2001 - December 2004 5-minute EUR/USD returns. The jump size $\kappa(s)$ is modeled as the the product between $\sigma(s)$ and a uniformly distributed random variable on $\sqrt{m}([-2,-1] \cup[1,2])$. The parameter $m$ determines the magnitude of the jumps. We set $m$ equal to either 0.1 (small jumps) or 1 (large jumps). For $m=0.1$, jumps cause about $20 \%$ of the daily variance of the returns. Finally, the jump occurrences $q(s)$ are specified as a Poisson process with on average one jump per day. These jump occurrences are either independent of $f$, either occur only in the 16 five-minute intervals for which volatility is periodically the lowest $(f<0.777)$ or in the 16 five-minute intervals for which volatility is periodically the highest $(f>1.3)$.

We simulate $K=500$ series of 500 days with 10 observations per 5-minute interval. Each day consists of 288 5-minute returns. The generated 5-minute return series is $r_{i}=p(i / 288)-p((i-1) / 288)$, with $i=1, \ldots, 288 \cdot 500$. The estimation of the periodicity factor is based on a local window length $\lambda$ equal to one day. We are interested in the effect of jumps on the bias and efficiency of the periodicity estimators. Recall that the non-parametric estimators use either the SD, ShortH or WSD as a scale estimator and that under the parametric approach, we have the choice between the OLS, ML or TML parameter estimators. This yields a total of 6 estimation methods. 
Figure 2: True periodicity function (dashed lines) versus the average estimate across the simulation (full line), for 6 different estimation procedures. The shaded region equals the range between the $2.5 \%$ and $97.5 \%$ quantiles. Jump occurrences are independent of $f$ and jumps are large $(m=1)$.
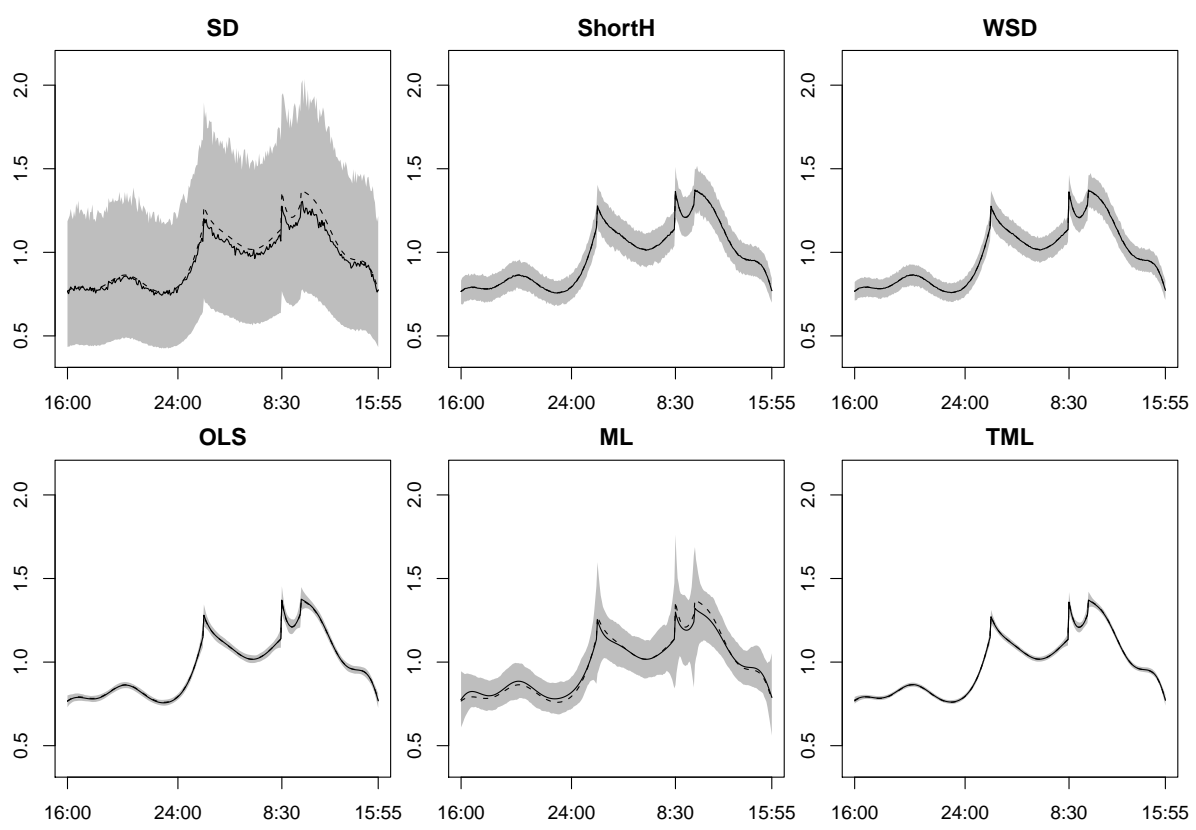

Bias. Figures 2-3 compare the true periodicity function $f$ with the average across the 500 simulations of the 6 estimators. The difference between both indicates the bias of the periodicity estimators caused by the price jumps. In Figure 2 jumps are large $(m=1)$ and their occurrences are uniformly distributed over the day. In Figure 3 jumps are small $(m=0.1)$ and their occurrences are concentrated on the intraday times for which $f<0.777$. We concentrate on these two cases because (holding the average number of jumps per day fixed to 1) the visual evidence of the bias of the non-robust estimators is more clear when jumps are large and/or jumps are concentrated on certain times of the day.

We see that jumps cause a large bias in the SD and ML estimators and, if jumps are concentrated on a part of the day, they also cause a bias in the OLS 
Figure 3: True periodicity function (dashed lines) versus the average estimate across the simulation (full line), for 6 different estimation procedures. The shaded region equals the range between the $2.5 \%$ and $97.5 \%$ quantiles. All jumps occur when $f<0.777$ and jumps are small $(m=0.1)$.
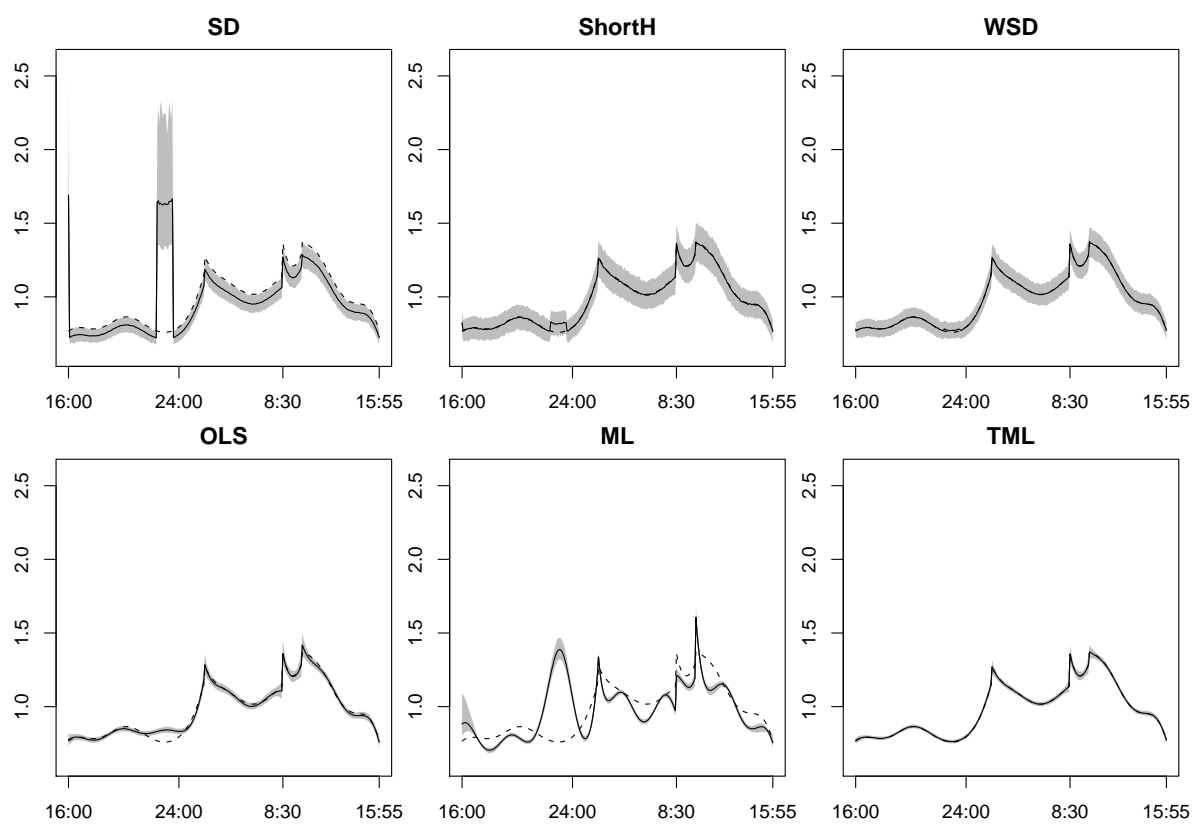

estimator. In Figure 3 we see that the SD, ML and OLS estimators overestimate the periodic component for the intervals with jumps and underestimate it for the intervals without jumps. For all types of jump processes considered, the WSD and TML estimators are the only estimators without visual evidence of a bias.

Efficiency. In Figures 2 and 3 we also report the $95 \%$ confidence bands. We see that, in the presence of jumps, the SD estimator has the largest standard error. More details on the relative efficiency of the estimators in the absence and presence of jumps are given in Table 1. This table reports for each of the six estimation procedures the Mean Absolute Error of the estimated value of the periodicity factors $\left(\mathrm{MAE}_{f}\right)$ and for the parametric estimators also the 
MAE of the estimated parameter vector $\left(\mathrm{MAE}_{\theta}\right)$. More precisely,

$$
\operatorname{MAE}_{f}=\frac{1}{K M T} \sum_{k=1}^{K} \sum_{i=1}^{M T}\left|\hat{f}_{i}^{k}-f_{i}\right| \text { and } \mathrm{MAE}_{\theta}=\frac{1}{K L} \sum_{k=1}^{K} \sum_{i=1}^{L}\left|\hat{\theta}_{i}^{k}-\theta_{i}\right|,
$$

where the number of components of $\theta$ equals $L=21$ and where $k=1, \ldots, K=$ 500 indicates the $k$ th simulation run.

The first panel in Table 1 reports the MAE of the estimators if there are no jumps in the price diffusion. We see that all parametric estimators have a much lower MAE than the non-parametric estimators. In the class of nonparametric estimators, the SD is the most efficient estimator. The ShortH has the largest MAE and the MAE of the WSD is between the MAE of the SD and ShortH. For the parametric estimators, the ML estimator is efficient. The TML estimator is only slightly less efficient than the ML estimator and the OLS estimator has the largest MAE of all parametric estimators. Note that the relative difference in the MAE for $\theta$ of the OLS estimator with respect to the ML and TML estimators is much larger than the relative difference of its MAE for $f$. This is possible, since it might be that two estimates for $\theta$ are quite different, but still result in a similar periodicity function.

The second and third panel of Table 1 report the MAE for a log-price diffusion with on average one jump per day. We see that the MAE of the $\mathrm{SD}$ in the presence of jumps is several times higher than its MAE in the absence of jumps. Also the ML estimator is extremely sensitive to jumps. The optimality of the ML estimator is thus restricted to the model without jumps. Since jumps do occur in practice, we recommend to use the TML estimator as an alternative. Note also that the MAE of the SD and TML estimators is especially large if jumps are large and/or concentrated on certain parts of the day.

The MAE for $f$ of the ShortH, WSD, OLS and TML periodicity estimators is little affected by the inclusion of jumps in the price process. The robustness of the OLS estimator is surprising at first sight, but it corroborates Martens et al. (2002)'s intuition that the log-transformation shrinks the outliers and makes the estimators based on a regression of the log absolute returns more 
Table 1: Mean absolute error (MAE) of the estimators for the periodicity factor and the associated parameter vector, for six different estimation methods. Jumps are small $(m=0.1)$ or large $(m=1)$ and their occurrences are either uniformly distributed over the day or concentrated on the parts of the day when volatility is periodically low $(f<0.777)$ or high $(f>1.333)$.

\begin{tabular}{|c|c|c|c|c|c|c|c|}
\hline \multirow[b]{2}{*}{ MAE } & & \multicolumn{3}{|c|}{ non-parametric estimation } & \multicolumn{3}{|c|}{ parametric estimation } \\
\hline & & SD & ShortH & WSD & OLS & ML & TML \\
\hline \multirow[t]{2}{*}{ No jumps } & $f$ & 0.025 & 0.039 & 0.030 & 0.011 & 0.006 & 0.007 \\
\hline & $\theta$ & & & & 0.197 & 0.010 & 0.011 \\
\hline \multicolumn{8}{|c|}{ One jump per day and jump occurrences are independent of $f$} \\
\hline \multirow[t]{2}{*}{ small jumps } & $f$ & 0.062 & 0.039 & 0.029 & 0.011 & 0.016 & 0.007 \\
\hline & $\theta$ & & & & 0.221 & 0.032 & 0.013 \\
\hline \multirow[t]{2}{*}{ large jumps } & $f$ & 0.218 & 0.039 & 0.029 & 0.011 & 0.056 & 0.007 \\
\hline & $\theta$ & & & & 0.213 & 0.142 & 0.019 \\
\hline \multicolumn{8}{|c|}{ One small jump per day and jumps occur only if } \\
\hline \multirow[t]{2}{*}{$f<0.777$} & $f$ & 0.110 & 0.041 & 0.030 & 0.020 & 0.111 & 0.007 \\
\hline & $\theta$ & & & & 0.257 & 0.192 & 0.012 \\
\hline \multirow[t]{2}{*}{$f>1.3$} & $f$ & 0.203 & 0.042 & 0.029 & 0.029 & 0.193 & 0.007 \\
\hline & $\theta$ & & & & 0.331 & 0.136 & 0.016 \\
\hline
\end{tabular}

robust to jumps. Note, however, that the TML estimator has a significantly lower MAE than the OLS estimator in all simulations considered here.

The main message of Figures 2 and 3 and of Table 1 is that the nonparametric WSD and parametric TML estimators have a relatively high efficiency in the absence of jumps. If jumps are present in the process, they are (for all configurations of the jump process) the most accurate of all nonparametric and parametric estimators considered, respectively. In all cases considered here, the TML estimator based on the correctly specified periodicity function is more efficient than the WSD estimator. For this reason, we recommend to estimate sufficiently smooth periodicity functions using the TML estimator based on a flexible parametric specification of the periodicity 
function.

\section{$3 \quad$ Intraday jump tests}

\subsection{The original test}

Andersen et al. (2007) and Lee and Mykland (2008) use the absolute value of the standardized return $\bar{r}_{i}=r_{i} / \hat{s}_{i}$ in (2.8) as a test statistic for the null hypothesis that $r_{i}$ is not affected by jumps. ${ }^{4}$ Denote the jump test statistic for $r_{i}$ by

$$
\mathrm{J}_{i}=\frac{\left|r_{i}\right|}{\hat{s}_{i}} .
$$

Under the BSMFAJ model and if $r_{i}$ is not affected by a jump, then for $\lambda \rightarrow 0$ and $\lambda / \Delta \rightarrow \infty$, the statistic $\mathrm{J}_{i}$ converges in distribution to the absolute value of a standard normal random variable (see Theorem 1 in Lee and Mykland, 2008).

A straightforward jump detection rule is that return $r_{i}$ is affected by a jump if $\mathrm{J}_{i}$ exceeds the $1-\alpha / 2$ quantile of the standard Gaussian distribution. This rule has a probability of type I error (detect that $r_{i}$ is affected by jumps, if in reality $r_{i}$ is not affected by jumps) equal to $\alpha$. But its disadvantage is that the expected number of false positives over the whole estimation sample becomes large. For example, with $M=288$ intraday returns per day and $\alpha=0.01$, one expects to detect about $0.01 \cdot 288 \approx 3$ jumps per day, even if no single jump has occurred. Lee and Mykland (2008) call these false positives "spurious jump detections".

Andersen et al. (2007) use a Bonferroni correction to control for the number of spurious jumps detected per day. As a rejection threshold, they propose to use the $\left[1+(1-\alpha)^{\Delta}\right] / 2$ quantile of the Gaussian distribution. Lee and Mykland

\footnotetext{
${ }^{4} \mathrm{~A}$ similar idea is pursued by Brownlees and Gallo (2006) for outlier detection in highfrequency return series, but they use the standard deviation as an estimator of local scale. The standard deviation has the disadvantage of not being robust to jumps. If jumps are present in the neighborhood of $i$, the return affected by jumps may, after standardization by the standard deviation, no longer be large.
} 
(2008) control for the size of the multiple jump tests using the extreme value theory result that the maximum of $n$ i.i.d. realizations of the absolute value of a standard normal random variable is asymptotically (for $n \rightarrow \infty$ ) Gumbel distributed (see e.g. Chapter 3 in Embrechts et al., 1999). More specifically, in the absence of jumps, the probability that the maximum of any set of $n$ J-statistics exceeds

$$
g_{n, \alpha}=-\log (-\log (1-\alpha)) b_{n}+a_{n}
$$

with $a_{n}=(2 \log n)^{1 / 2}-[\log \pi+\log (\log n)] /\left[2(2 \log n)^{1 / 2}\right]$ and $b_{n}=1 / \sqrt{2 \log n}$, is about $\alpha$. Lee and Mykland (2008)'s proposal is that all returns for which the $\mathrm{J}$ test statistic exceeds this threshold $g_{n, \alpha}$ should be declared as being affected by jumps. In the sequel of the paper, we use $n=1 / \Delta=288$. This corresponds to testing for the joint null hypothesis of no jumps over one day. We set $\alpha=1 \%$. For these values of $n, \Delta$ and $\alpha$, Andersen et al. (2007)'s and Lee and Mykland (2008)'s threshold equals 4.139 and 4.305, respectively.

\subsection{The filtered test}

The original test in (3.1) assumes that the spot volatility $\sigma(s)$ is approximately constant over the local window used to compute $\hat{s}_{i}$. This is a reasonable assumption for short local windows such as 30 minutes. However, if returns are sampled at frequencies of one hour, 30 minutes, 15 minutes or 5 minutes, Lee and Mykland (2008) recommend to use local windows containing 78, 110, 156 or 270 observations, respectively. ${ }^{5}$ This corresponds to local windows of at least $90 \%$ of a day. Also Andersen et al. (2007) use local windows of one day in their application on the 2-min transaction returns from the S\&P 500 futures contract. For such long windows, the assumption of constant volatility is at odds with the overwhelming empirical evidence that the intraday variation in market activity causes intraday volatility to be strongly time-varying and even displays discontinuities (Taylor, 2004). Consequently $\hat{s}_{i}$ does not estimate the

\footnotetext{
${ }^{5}$ These numbers correspond to the smallest number of observations for which jumps will have a negligible effect on $\hat{s}_{i}$ (Lee and Mykland, 2008).
} 
volatility of $r_{i}$, but the average level of volatility of the returns in the local window of $r_{i}$.

Andersen et al. (2007) recognize this and as a robustness check, they verify their empirical results using the returns standardized by a periodicity estimate that is similar to the SD estimator. The test statistic based on the original J statistic divided by a periodicity estimate is called the "filtered J test statistic". Andersen et al. (2007) use the filtered J test statistic based on the SD periodicity estimator

$$
\mathrm{FJ}_{i}^{\mathrm{SD}}=\frac{\left|r_{i}\right|}{\hat{f}_{i}^{\mathrm{SD}} \hat{s}_{i}}
$$

We propose to use a filtered J test based on the robust WSD and TML periodicity estimates, i.e.

$$
\mathrm{FJ}_{i}^{\mathrm{WSD}}=\frac{\left|r_{i}\right|}{\hat{f}_{i}^{\mathrm{WSD}} \hat{s}_{i}} \quad \text { and } \quad \mathrm{FJ}_{i}^{\mathrm{TML}}=\frac{\left|r_{i}\right|}{\hat{f}_{i}^{\mathrm{TML}} \hat{s}_{i}} .
$$

We have the following result (proof is in appendix).

Result 5 Under the BSMFAJ model with spot volatility process satisfying Assumption 1 and with jump occurrence process satisfying Assumption 2, and if there is no jump at time $s$, we have that the statistics $F J_{i}^{W S D}$ and $F J_{i}^{T M L}$ converge in distribution to the absolute value of a standard normal random variable for $\lambda \rightarrow 0, \lambda / \Delta \rightarrow \infty$ and $T \rightarrow \infty$.

In the absence of a periodic variation of volatility in the local window, the original $\mathrm{J}$ test statistic is of course to be preferred over its filtered counterpart, because of the uncertainty in the estimated periodicity factor. However, for sufficiently long local windows such as when $\lambda$ is equal to one day, there is a strong periodic intrawindow variation of volatility. Then intraday jump detection using the filtered $\mathrm{J}$ test statistic will yield more accurate results.

\subsection{Simulation study}

We now compare testing for jumps using the original and filtered $\mathrm{J}$ test statistics by means of a simulation study. The implementation is based on a local 
window length $\lambda$ equal to one day. We use the rejection threshold in (3.2) with $\alpha=0.01$ and $n=288$ (the number of 5-minute intervals per day of 24 hours). This means that the returns for which the $\mathrm{J}$ test statistic exceeds 4.305 are identified as being affected by jumps. We generate $K=500$ series of 500 days of 5-minute returns from a process that is the same as in Subsection 2.5, except that the specification of the periodicity function is more simple, namely:

$f(\tau(s))=0.447 \cdot I(\tau(s) \leq 1 / 3)+I(1 / 3<\tau(s) \leq 2 / 3)+1.342 \cdot I(2 / 3<\tau(s) \leq 1)$.

Under this specification, the intraday volatility is periodically low in the first 8 hours of the day and periodically high in the last 8 hours of the day.

Like Andersen et al. (2007) and Lee and Mykland (2008) we use the proportion of spuriously detected jumps and proportion of actual jumps that have been detected with success as indicators of the size and power of the test. Andersen et al. (2007) call these statistics "effective size" and "effective power", respectively. They are reported in Table 2 for the case of no jumps and 1 jump per day. The jumps we consider are similar as in Table 1: they are either small $(m=0.1)$ or large $(m=1)$ and are either uniformly distributed over the day or concentrated on the parts where volatility is periodically low or high. The effective size and power are reported as a function of the value of the periodicity function.

Effective size. The first panel in Table 2 reports the proportion of returns for which a jump has been detected by the original and the filtered $J$ tests, if in reality there are no jumps in the process. Recall that a jump is detected when the original or filtered $\mathrm{J}$ statistics exceed 4.305. Asymptotically (for $\lambda \rightarrow 0$ and $\lambda / \Delta \rightarrow \infty)$ these J statistics converge to the absolute value of a standard normal random variable for which the probability to be larger than 4.305 equals 1.7e-5. Because we take $\lambda$ equal to one day and because of the time-varying volatility the actual effective size is slightly higher. Note that the original $\mathrm{J}$ test has an important size distortion for $f \neq 1$. If $f=0.447$, it detects no spurious jumps at all and if $f=1.342$ then $0.14 \%$ of all returns are (spuriously) identified as being affected by jumps. The original J test thus underdetects 
(overdetects) jumps if the value of the periodicity function is low (high). The differences in effective size of the filtered $\mathrm{J}$ test are economically insignificant with respect to the variation in effective size observed for the $J$ test. In the case of 500 days of 2885 -minute returns, the J statistic detects on average between $0(f=0.447)$ and $202(f=1.342)$ spurious jumps, while the filtered J tests detect only between $3\left(\mathrm{FJ}^{\mathrm{SD}} ; f=1.342\right)$ and $7\left(\mathrm{FJ}^{\mathrm{WSD}} ; f=0.447\right)$ spurious jumps.

In panel 2 of Table 2 we report the effective size of the tests in the presence of jumps. Note that also in the presence of jumps, the effective size of the $\mathrm{J}$ test is highly dependent on the value of the periodicity factor. For all types of jumps considered, the effective size of the filtered J test based on the WSD or TML estimators has the correct order of magnitude (1e-5). In the case of large jumps $(m=1)$, the effective size of the filtered $\mathrm{J}$ test based on the SD estimator is too large. It is about $2.4 \mathrm{e}-3$, which implies on average 345 spurious jump detections per 500 days. This is due to the bias and inefficiency of the SD periodicity estimator in the presence of large jumps (see Figure 2 and Table 1).

Effective power. The detection of large jumps is only marginally affected by the presence of periodicity in the spot volatility. Let us therefore focus on the power to detect small jumps, which are the most difficult to detect. In panel 2 of Table 2 we see that the original J test detects only $18 \%$ of all jumps if jumps are small $(m=0.1)$ and occur when volatility is periodically low $(f=0.447)$. The robustly filtered $\mathrm{J}$ tests detect more than $96 \%$ of the actual jumps in this case. Figure 3 illustrates that if jumps only occur when volatility is periodically low, the SD periodicity has a large upward bias. Because of this bias, the power of the filtered J test based on the SD is only $88 \%$ in the case of small jumps occurring only when volatility is periodically low, while for the robust filtered $\mathrm{J}$ test it is $98 \%$. 
Table 2: Effective size and effective power for the original and filtered $\mathrm{J}$ tests with rejection threshold equal to 4.305 as a function of the periodicity factor. Jumps are small $(m=0.1)$ or large $(m=1)$ and their occurrences are either uniformly distributed over the day or concentrated on the parts of the day when volatility is periodically low $(f=0.447)$ or high $(f=1.342)$.

\begin{tabular}{|c|c|c|c|c|c|c|c|c|}
\hline & \multicolumn{4}{|c|}{ effective size } & \multicolumn{4}{|c|}{ effective power } \\
\hline & $\mathrm{J}$ & $\mathrm{FJ}^{\mathrm{SD}}$ & $\mathrm{FJ}^{\mathrm{WSD}}$ & $\mathrm{FJ}^{\mathrm{TML}}$ & $\mathrm{J}$ & $\mathrm{FJ}^{\mathrm{SD}}$ & FJWSD & $\mathrm{FJ}^{\mathrm{TML}}$ \\
\hline \multicolumn{9}{|l|}{ No jumps } \\
\hline$f=0.447$ & 0 & $3.0 \mathrm{e}-5$ & $4.6 \mathrm{e}-5$ & $3.5 \mathrm{e}-5$ & & & & \\
\hline$f=1$ & $2.4 \mathrm{e}-5$ & $2.1 \mathrm{e}-5$ & $3.0 \mathrm{e}-5$ & $2.2 \mathrm{e}-5$ & & & & \\
\hline$f=1.342$ & $1.4 \mathrm{e}-3$ & $1.9 \mathrm{e}-5$ & $2.6 \mathrm{e}-5$ & $2.1 \mathrm{e}-5$ & & & & \\
\hline \multicolumn{9}{|c|}{ One small jump per day and jump occurrences are independent of $f$} \\
\hline$f=0.447$ & 0 & $6.5 \mathrm{e}-5$ & $5.4 \mathrm{e}-5$ & $6.0 \mathrm{e}-5$ & 0.1760 & 0.9684 & 0.9762 & 0.9802 \\
\hline$f=1$ & $3.1 \mathrm{e}-5$ & $4.9 \mathrm{e}-5$ & $3.9 \mathrm{e}-5$ & $4.7 \mathrm{e}-5$ & 0.9758 & 0.9692 & 0.9751 & 0.9799 \\
\hline$f=1.342$ & $1.8 \mathrm{e}-3$ & $4.2 \mathrm{e}-5$ & $2.9 \mathrm{e}-5$ & $3.6 \mathrm{e}-5$ & 0.9972 & 0.9681 & 0.9732 & 0.9785 \\
\hline \multicolumn{9}{|c|}{ One large jump per day and jump occurrences are independent of $f$} \\
\hline$f=0.447$ & 0 & $2.5 \mathrm{e}-3$ & $4.2 \mathrm{e}-5$ & $3.2 \mathrm{e}-5$ & 0.9982 & 0.9996 & 0.9996 & 0.9996 \\
\hline$f=1$ & $2.2 \mathrm{e}-5$ & $2.4 \mathrm{e}-3$ & $2.7 \mathrm{e}-5$ & $2.2 \mathrm{e}-5$ & 0.9996 & 0.9996 & 0.9996 & 0.9996 \\
\hline$f=1.342$ & $1.4 \mathrm{e}-3$ & $2.4 \mathrm{e}-3$ & $2.3 \mathrm{e}-5$ & $1.8 \mathrm{e}-5$ & 0.9997 & 0.9996 & 0.9996 & 0.9996 \\
\hline \multicolumn{9}{|c|}{ One small jump per day and jumps only occur if $f=0.447$} \\
\hline$f=0.447$ & 0 & $2.9 \mathrm{e}-6$ & $6.7 \mathrm{e}-5$ & $5.5 \mathrm{e}-5$ & 0.2075 & 0.8773 & 0.9783 & 0.9785 \\
\hline \multicolumn{9}{|c|}{ One small jump per day and jumps only occur if $f=1.342$} \\
\hline$f=1.342$ & $1.5 \mathrm{e}-3$ & $1.7 \mathrm{e}-5$ & $2.6 \mathrm{e}-5$ & $2.1 \mathrm{e}-5$ & 0.9953 & 0.9264 & 0.9646 & 0.9658 \\
\hline
\end{tabular}




\section{Illustration on 5-min EUR/USD returns}

Like Andersen and Bollerslev (1997, 1998b), Martens et al. (2002) and Taylor and $\mathrm{Xu}$ (1997), we study the intraweek periodicity in the volatility of 5-minute EUR/USD returns. Our data set, provided by Olsen and Associates, ranges from January 2001 - December 2004 and time is expressed in Eastern Standard Time (EST), taking into account the daylight saving time shifts in Europe and America. More details on this data set can be found in Beine et al. (2006). We consider a local window length $\lambda$ equal to one day and thus assume that the intraday variation in volatility is mainly due to a periodic function $f$ that is a deterministic function of the intraweek time. ${ }^{6}$

Non-parametric periodicity estimates. Figure 4 plots the SD, ShortH and WSD-based non-parametric estimates of the intraweek periodic component in the volatility of 5 -minute EUR/USD returns. The first value is the periodicity factor for the 5-minute return on Sunday evening at 16:00-16:05 EST. We see that for most intervals, the classical and robust periodicity estimates closely resemble each other. Two notable exceptions are the 8:30-35 and 10:00-10:05 EST intervals during which the most important macroeconomic news are released (Ederington and Lee, 1993) and Sunday evening. It is likely that these differences are due to jumps.

Consider e.g. the 8:30-8:35 interval on Friday morning. According to the SD estimator, the periodicity factor is around 5, while according to the robust estimators it is around 3. Such a large difference between these estimators can only be due to the presence of jumps in the data. This mirrors the fact that in this interval many macroeconomic news are released and that these news releases are often associated with jumps. Note however that also according to the robust estimators, there is a sharp increase in the periodic component of

\footnotetext{
${ }^{6}$ Alternative approaches for analyzing such time series are to condition not only on intraweek time, but also on macroeconomic news announcements (Andersen and Bollerslev, 1997) or to allow the periodicity in intraday volatility to be stochastic (Beltratti and Morana, 2001).
} 
intraday volatility at the time of the macroeconomic news releases. ${ }^{7}$

There is also a noticeable difference in the Sunday evening periodicity estimates. The first periodicity factor of the weekly cycle equals 0.51 according to the SD estimator and only 0.33 according to the WSD estimator. Again, this difference must be due to jumps. The presence of many jumps in these intervals is plausible, since at these times there are relatively few trades on the FX market and price volatility is low. Since the market is then also the least liquid, it is easier for a trader to create jumps in the price process. These jumps inflate the SD periodicity estimator.

Parametric periodicity estimates. Figure 5 plots the parametric estimates of the intraweek periodicity factors obtained using the OLS, ML and TML parameter estimates. These estimates are based on specifying the log of the intraweek periodicity factor as a function of the Time of the Day interval $\mathrm{ToD}_{i}=1, \ldots, 288$ and Day of the Week $\mathrm{DoW}_{i}=1, \ldots, 5$ corresponding to the time point $t_{i}=i \Delta$. We use a specification that is similar to the one proposed by Andersen and Bollerslev (1997),

$$
\begin{aligned}
\log f_{i} & =\theta_{1} \frac{\mathrm{ToD}_{i}}{M_{1}}+\theta_{2} \frac{\mathrm{ToD}_{i}^{2}}{M_{2}} \\
& +\sum_{j=1}^{6} \theta_{3+j} \cos \left(\operatorname{ToD}_{i} \frac{2 \pi j}{M}\right)+\sum_{j=1}^{4} \theta_{9+j} \sin \left(\mathrm{ToD}_{i} \frac{2 \pi j}{M}\right) \\
& +\left(\theta_{14}+\theta_{15} \mathrm{ToD}_{i}\right) I\left(\mathrm{ToD}_{i} \leq 36\right) I\left(\mathrm{DoW}_{i}=1\right) \\
& +\sum_{d=1}^{5} \sum_{b=1}^{3} \theta_{16+3(d-1)+b} P_{b}\left(\mathrm{ToD}_{i} ; j\right) I\left(\mathrm{ToD}_{i} \geq j_{1}\right) I\left(\mathrm{DoW}_{i}=d\right) \\
& +\sum_{d=2}^{5} \sum_{a=2}^{3} \sum_{b=1}^{3} \theta_{31+6(d-2)+3(a-2)+b} P_{b}\left(\mathrm{ToD}_{i} ; j_{a}\right) I\left(\mathrm{ToD}_{i} \geq j_{a}\right) I\left(\mathrm{DoW}_{i}=d\right)
\end{aligned}
$$

where $M_{1}=M^{-1} \sum_{i=1}^{M} i=(M+1) / 2$ and $M_{2}=M^{-1} \sum_{i=1}^{M} i^{2}=\left(2 M^{2}+\right.$ $3 M+1) / 6$ are normalizing constants. The unknown parameter vector $\theta$ has

\footnotetext{
${ }^{7}$ Macro-economic news releases often have a monthly frequency. Since the release of different types of news are spread over the different weeks of the month (see e.g. Table 2 in Andersen et al., 2003), the release of a news on Friday 8:30 has a weekly frequency and therefore leads to a sharp increase in the periodic component of intraday volatility of the Friday 8:30-8:35 return.
} 
Figure 4: Non-parametric SD, ShortH and WSD estimates of the intraweek periodicity in the volatility of 5-minute EUR/USD returns. Time is expressed in EST.
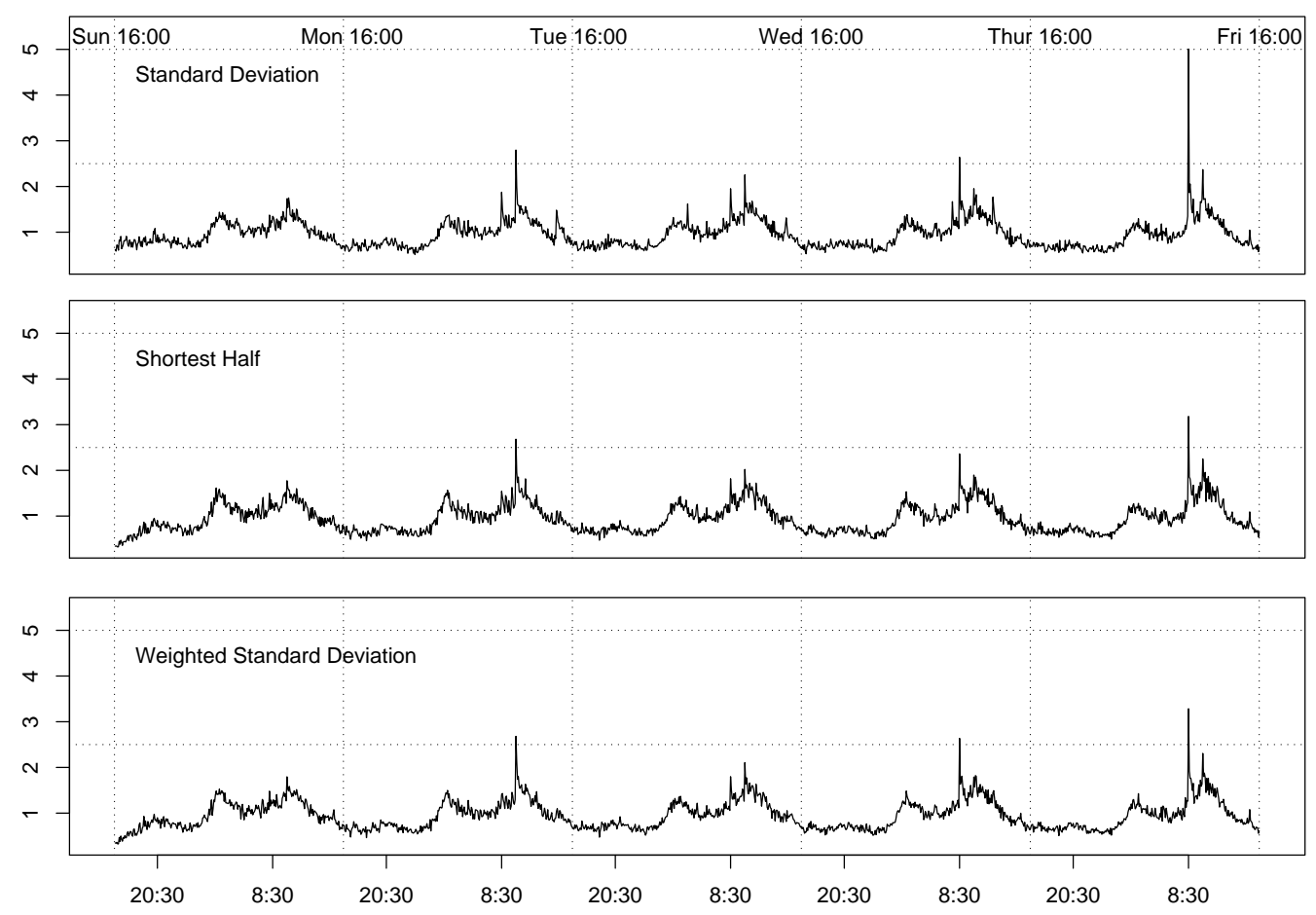

54 components. The last terms in the specification for $\log f_{i}$ are $b$ th order Almond polynomials of $t$ centered at $j_{a}$ and $b=1,2$ or 3 ,

$$
P_{b}\left(t ; j_{a}\right)=\left[1-\left(\left(t-j_{a}\right) / M\right)^{b}\right]\left(t-j_{a}\right)^{3-b} .
$$

We consider 3 centering points: $j_{1}, j_{2}$ and $j_{3}$, corresponding to the 2:30-2:35, 8:30-8:35 and 10:00-10:05 time intervals, respectively. The 2:30-2:35 polynomial is needed to accommodate for the increase in activity due to the opening of the European markets. The 8:30-8:35 and 10:00-10:05 polynomials are included to accommodate the increase in the periodic component of volatility due to the numerous releases of macroeconomic news in these intervals (Ederington and Lee, 1993). These polynomials have previously been used for this by Andersen et al. (2000) and Bollerslev et al. (2000), among others. 
Figure 5: Parametric OLS, ML and TML estimates of the intraweek periodicity in the volatility of 5-minute EUR/USD returns. The WSD-based nonparametric estimates (dashed line) are reported as benchmark. Time is expressed in EST.
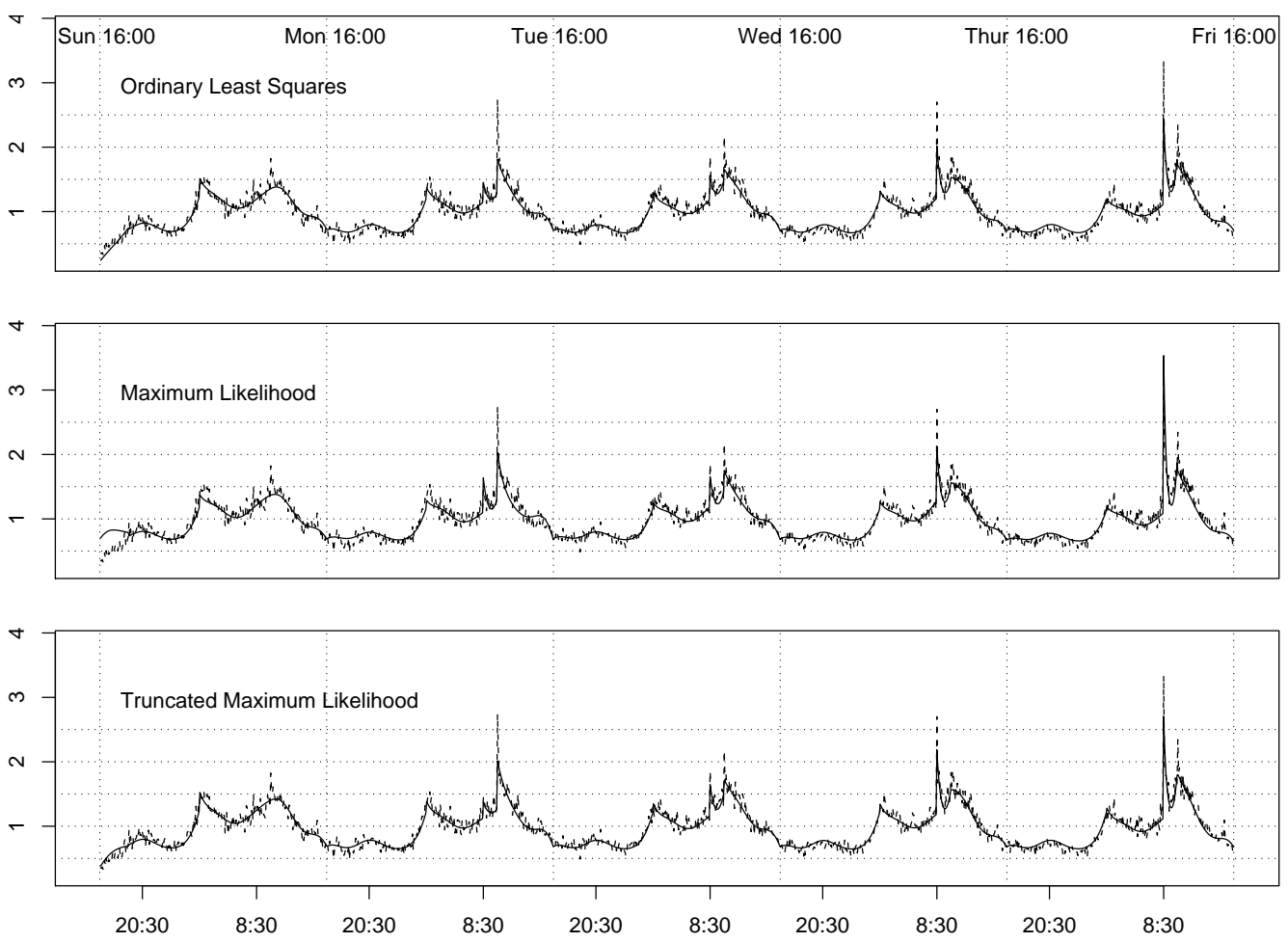

Figure 5 compares the parametric periodicity estimates with the WSDbased estimate. We see that, on Sunday evening, the OLS and TML periodicity estimators are close to the WSD estimate, and that the ML estimate is completely different than the robust estimates. The peaks in the OLS and TML parameter estimates are always smaller than the peaks in the WSD estimate. Note also that the OLS and TML periodicity estimates resemble each other. As explained by Martens et al. (2002), the log-transformation attenuates the effect of jumps on the estimator and thus makes it more robust to jumps.

Intraday jump detection. In Table 3 we report the proportion of returns 
affected by jumps according to the original and the filtered $\mathrm{J}$ tests (using either the WSD or TML periodicity estimates). The rejection threshold equals 4.305, which corresponds to the threshold in (3.2) with $\alpha=0.01$ and $n=288$. We find that all three considered test statistics detect that between $.36 \%$ and $.40 \%$ returns are affected by jumps. However, for more than half of the detected jumps, the original and filtered $\mathrm{J}$ tests do not agree.

Let us now study in more detail in which 5-minute intervals of the day the jumps are detected. Table 3 reports details on this. We find that in the high periodicity intervals $\left(\hat{f}_{i}^{\mathrm{WSD}}>1.3\right)$, the original $\mathrm{J}$ test detects more than 5 times more jumps than do the filtered $J$ tests. The opposite is true for the low periodicity intervals $\left(\hat{f}_{i}^{\mathrm{WSD}}<0.77\right)$ for which the filtered $\mathrm{J}$ tests detect more than 6 times more jumps. Note also that for most intervals, the proportion of detected jumps by the filtered J test using the TML estimator is between the proportion of detected jumps by the $\mathrm{J}$ test and the filtered $\mathrm{J}$ test using the WSD. This is because the range of the WSD estimate of the periodicity function is larger than the range of the TML estimate (Figure 5).

Price jumps are caused by a sudden release of news or arrival of orders. In the EUR/USD market, most macroeconomic news are released on TuesdayFriday 8:30-8:35 EST and Monday-Friday 10:00-10:05 EST. These are also the time intervals for which intraday volatility is periodically high. In Table 3 we report the proportion of detected jumps for these periods. We see that the filtered J tests detect significantly less jumps for these intervals. For Friday 8:30-8:35 EST, and Wednesday-Thursday 10:00-10:05, both the original and filtered $\mathrm{J}$ tests find that more than $5 \%$ of the returns correspond to jumps. According to the original J test 25\% of all returns in the Friday 8:30-8:35 EST interval, are affected by jumps and for the filtered $\mathrm{J}$ tests it is $7 \%$.

A second cause of price jumps is a sudden arrival of orders. These arrivals are more likely to create jumps at a time of low liquidity in the market (Farmer et al., 2004), such as on Sunday evening and lunch time of the Tokyo market, when the liquidity on the FX markets is periodically low. We see that for these intervals the filtered $J$ tests detect several times more jumps than the original $\mathrm{J}$ test. 
Table 3: Proportion of 5-minute EUR/USD returns for which the original and filtered J tests, with rejection threshold equal to 4.305, detect jumps.

\begin{tabular}{|lccc|}
\hline & $\mathrm{J}$ & FJ JSD $^{\text {FJ }}$ & FJL $^{\text {TML }}$ \\
\hline All intervals & .0040 & .0039 & .0036 \\
Intervals with $\hat{f}_{i}^{\text {WSD }}>1.3$ & .0152 & .0020 & .0030 \\
Tues, 8:30-8:35 & .0476 & .0265 & .0265 \\
Wed, 8:30-8:35 & .0645 & 0 & .0108 \\
Thur, 8:30-8:35 & .1243 & 0 & .0108 \\
Fri, 8:30-8:35 & .2473 & .0714 & .0714 \\
Mon, 10:00-10:05 & .0281 & 0 & .0112 \\
Tues, 10:00-10:05 & .1217 & 0 & .0212 \\
Wed, 10:00-10:05 & .0591 & .0054 & .0215 \\
Thu, 10:00-10:05 & .0378 & .0054 & .0108 \\
Fri, 10:00-10:05 & .0989 & 0 & .0220 \\
Intervals with 0.77 $\leq \hat{f}_{i}^{\text {WSD }} \leq 1.3$ & .0032 & .0029 & .0027 \\
Intervals with $\hat{f}_{i}^{\text {WSD }}<0.77$ & .0008 & .0059 & .0052 \\
Sunday Evening $(16: 00-19: 00)$ & .0022 & .0275 & .0362 \\
Tokyo Lunch $(23: 00-00: 45)$ & .0004 & .0040 & .0022 \\
\hline
\end{tabular}

\section{Conclusion}

In this paper, we propose the weighted standard deviation and truncated maximum likelihood periodicity estimators as an alternative for Taylor and Xu (1997)'s non-parametric and Andersen and Bollerslev (1997)'s parametric periodicity estimators, respectively. The new estimators are robust to price jumps. For sufficiently smooth periodicity functions, we recommend the truncated maximum likelihood estimator based on Andersen and Bollerslev (1997)'s flexible specification for the periodicity function, because of its high efficiency both in the absence and presence of jumps. We compare the classical and robust periodicity estimators for the 5-minute EUR/USD returns. We find large differences between the classical and robust periodicity estimators 
for the Sunday evening trading intervals and the intervals during which usually macroeconomic news are released.

We also show that the robust periodicity estimates can be used to increase the accuracy of intraday jump detection methods. It seems that filtering matters especially for the size of the test: the original test overdetects jumps at times of periodically low intraday volatility and underdetect jumps at times of periodically high intraday volatility. Filtering is also important to increase the power of the test to detect small jumps at times of periodically low volatility, such as on Sunday evening and at the Tokyo lunch time. Using the filtered jump test statistics, we detect significantly less jumps for the intraday intervals during which macroeconomic news are released.

The robust periodicity estimators can yield very different estimates than the classical ones in the presence of jumps. We illustrated this for the 5-minute EUR/USD returns, but we expect similar differences for other series. A topic of future research is to explore the potential gains in accuracy of using these robust periodicity estimates for forecasting intraday volatility (Taylor and $\mathrm{Xu}$, 1997) or for bootstrapping realized volatility (Gonçalves and Meddahi, 2008).

\section{Appendix}

The proofs are based on the following lemma.

Lemma 1 Under the BSMFAJ model with spot volatility process satisfying Assumption 1, we have that, if there is no jump at $s, \bar{r}(s)$ converges in distribution to a normal random variable with mean zero and variance $f^{2}(\tau(s))$ for $\lambda \rightarrow 0$ and $\lambda / \Delta \rightarrow \infty$.

Proof of Lemma 1. The lemma follows directly from the consistency of the realized bipower variation for the integrated variance of a BSMFAJ process and from Assumption 1.

Proof of Result 1. This proposition follows from Lemma 1 and from the consistency of the sample standard deviation, shortest half dispersion and the 
outlyingness weighted standard deviation (see Boudt et al., 2008b) for the standard deviation of an i.i.d. sample of standard normal random variables.

Proof of Result 2. If the number of jumps is finite, the proportion of returns affected by jumps goes to zero when $\Delta \rightarrow 0$. By the bounded influence function property of the shortest half dispersion and the weighted standard deviation based on the shortest half dispersion (Rousseeuw and Leroy, 1988; Boudt et al., $2008 \mathrm{~b}$ ), the effect of infinitesimal contamination by jumps on these estimators is asymptotically negligible.

Proof of Result 3. Denote $l$ and $u$ the values of $z$ for which $\rho^{\mathrm{ML}}(z)$ equals $\rho^{\mathrm{ML}}(q)$. Result 1 ensures that, for $s=i \Delta$,

$$
\operatorname{plim}_{\Delta \rightarrow 0} \hat{f}^{\mathrm{WSD}}(\tau(s))=f(\tau(s))=\exp \left(\theta_{*}^{\prime} x(\tau(s))\right) .
$$

Let $H$ be the distribution of the design variables $x$. Then it follows that the objective function in (2.20) converges in probability to

$$
\phi(\theta)=\iint_{l}^{u} \rho^{\mathrm{ML}}\left(z+\theta_{*}^{\prime} x-\theta^{\prime} x\right) g(z) d z d H(x),
$$

with $g$ defined in $(2.15)$. Since $\rho^{\mathrm{ML}}(z)$ is a convex function, there is a unique minimum and hence, for establishing consistency, it is sufficient to prove that $\theta_{*}$ is a critical value of $\phi(\theta)$. The remainder of the proof follows from Remark 5 in Marazzi and Yohai (2004). The first order condition is that

$$
\begin{aligned}
\left.\frac{\partial \phi(\theta)}{\partial \theta}\right|_{\theta=\theta_{*}} & =-\iint_{l}^{u} \frac{d \rho^{\mathrm{ML}}(z)}{d z} x g(z) d z d H(x) \\
& =-[g(u)-g(l)] \int x d H(x)=0 .
\end{aligned}
$$

The latter equality follows from $\rho^{\mathrm{ML}}(z)=\log g(z)$ which implies that $d \rho^{\mathrm{ML}}(z) / d z=$ $g^{-1}(z) d g(z)$ and $g(u)=g(l)$.

Proof of Result 4. By the consistency of $\hat{f}_{i}^{\mathrm{WSD}}$ under the BSMFAJ model (Result 2), all returns affected by jumps have a residual $e_{i}^{\text {wSD }}$ with likelihood $\rho^{\mathrm{ML}}\left(e_{i}^{\mathrm{WSD}}\right)$ above the threshold $\rho^{\mathrm{ML}}(q)$ and receive a zero weight in the estimation. Jumps therefore have no effect on the TML-estimator, and its consistency 
for $\theta_{*}$ under the BSMFAJ model follows from its consistency for $\theta_{*}$ under the BSM model (Result 3).

Proof of Result 5. By Lemma 1, $|\bar{r}(s)|$ converges in distribution to the absolute value of normal random variable with mean zero and variance $f^{2}(\tau(s))$. By Result 4, $\hat{f}^{\mathrm{WSD}}(\tau(s))$ and $\hat{f}^{\mathrm{TML}}(\tau(s))$ converge in probability to $f(\tau(s))$. Hence, by Cramér's theorem, $|\bar{r}(s)| / \hat{f}^{\mathrm{WSD}}(\tau(s))$ and $|\bar{r}(s)| / \hat{f}^{\mathrm{TML}}(\tau(s))$ converge in distribution to the absolute value of a standard normal random variable.

\section{References}

Andersen, T., T. Bollerslev, and J. Cai (2000). Intraday and interday volatility in the Japanese stock market. Journal of International Financial Markets, Institutions and Money 10, 107-130.

Andersen, T., T. Bollerslev, and A. Das (2001). Variance-ratio statistics and high-frequency data: testing for changes in intraday volatility patterns. Journal of Finance 56, 305-327.

Andersen, T., T. Bollerslev, and F. Diebold (2007). Roughing it up: including jump components in the measurement, modelling and forecasting of return volatility. The Review of Economics and Statistics 89, 701-720.

Andersen, T., T. Bollerslev, and D. Dobrev (2007). No-arbitrage semimartingale restrictions for continous-time volatility models subject to leverage effects, jumps and i.i.d. noise: Theory and testable distributional implications. Journal of Econometrics 138, 125-180.

Andersen, T. G. and T. Bollerslev (1997). Intraday periodicity and volatility persistence in financial markets. Journal of Empirical Finance 4, 115-158.

Andersen, T. G. and T. Bollerslev (1998a). Answering the skeptics: yes, standard volatility models do provide accurate forecasts. International Economic Review 39, 885-905. 
Andersen, T. G. and T. Bollerslev (1998b). Deutsche Mark-Dollar volatility: intraday activity patterns, macroeconomic announcements, and longer run dependencies. Journal of Finance 53, 219-265.

Andersen, T. G., T. Bollerslev, F. Diebold, and C. Vega (2003). Micro effects of macro announcements: real-time price discovery in foreign exchange. American Economic Review 93, 38-67.

Barndorff-Nielsen, O. and N. Shephard (2004). Power and bipower variation with stochastic volatility and jumps. Journal of Financial Econometrics 2, $1-37$.

Beine, M., J. Lahaye, S. Laurent, C. Neely, and F. Palm (2006). Central bank intervention and exchange rate volatility, its continuous and jump components. International Journal of Finance and Economics 12, 201-223.

Beltratti, A. and C. Morana (2001). Deterministic and stochastic methods for estimation of intra-day seasonal components with high frequency data. Economic notes 30, 205-234.

Bollerslev, T., J. Cai, and F. M. Song (2000). Intraday periodicity, long memory volatility, and macroeconomic announcement effects in the US treasury bond market. Journal of Empirical Finance 7, 37-55.

Bos, C. S. (2008). Model-based estimation of high frequency jump diffusions with microstructure noise and stochastic volatility. Mimeo.

Boudt, K., C. Croux, and S. Laurent (2008a). Asymptotic variance of the Realized Outlyingness Weighted Variance. Technical report. Available at www.econ.kuleuven.be/kris.boudt/public.

Boudt, K., C. Croux, and S. Laurent (2008b). Outlyingness weighted quadratic covariation. Mimeo.

Brownlees, C. and G. Gallo (2006). Financial econometric analysis at ultrahigh frequency: Data handling concerns. Computational Statistics \& Data Analysis 51, 2232-2245. 
Ederington, L. H. and J. H. Lee (1993). How markets process information: news releases and volatility. Journal of Finance 158, 1161-1191.

Embrechts, P., C. Klüpelberg, and T. Mikosch (1999). Modelling Extremal Events for Insurance and Finance. Application of Mathematics. SpringerVerlag.

Farmer, J. D., L. Gillemot, F. Lillo, S. Mike, and A. Sen (2004). What really causes large price changes? Quantitative Finance 4, 383-397.

Gonçalves, S. and N. Meddahi (2008). Bootstrapping realized volatility. Econometrica, forthcoming.

Goodhart, C. A. and M. O'Hara (1997). High frequency data in financial markets: Issues and applications. Journal of Empirical Finance 4, 73-114.

Ito, T., R. K. Lyons, and M. T. Melvin (1998). Is there private information in the FX market? The Tokyo experiment. Journal of Finance 53, 1111-1130.

Lahaye, J., S. Laurent, and C. Neely (2007). Jumps, cojumps and macro announcements. Mimeo.

Lee, S. S. and P. A. Mykland (2008). Jumps in financial markets: a new nonparametric test and jump dynamics. Review of Financial studies, forthcoming.

Marazzi, A. and V. J. Yohai (2004). Adaptively truncated maximum likelihood regression with asymmetric errors. Journal of Statistical Planning and Inference 122, 271-291.

Maronna, R. A., D. R. Martin, and V. J. Yohai (2006). Robust Statistics: Theory and Methods. Wiley.

Martens, M., Y.-C. Chang, and S. J. Taylor (2002). A comparison of seasonal adjustment methods when forecasting intraday volatility. Journal of Financial Research 25, 283-299. 
Martin, R. and R. Zamar (1993). Bias robust estimates of scale. The Annals of Statistics 21, 991-1017.

Rousseeuw, P. and A. Leroy (1988). A robust scale estimator based on the shortest half. Statistica Neerlandica 42, 103-116.

Taylor, N. (2004). Modeling discontinuous periodic conditional volatility: evidence from the commodity futures market. Journal of Futures Markets 9, 805-834.

Taylor, S. J. and X. Xu (1997). The incremental volatility information in one million foreign exchange quotations. Journal of Empirical Finance 4, $317-340$.

Zhang, L., P. A. Mykland, and Y. Ait-Sahalia (2005). A tale of two time scales: Determining integrated volatility with noisy high-frequency data. Journal of the American Statistical Association 100, 1394-1411. 